\title{
Filigrane
}

Écoutes psychothérapiques

\section{La supervision en psychanalyse. Mutualité, assymétrie et refusement. Entrevue avec Lise Monette et Bernadette Tanguay}

\section{France Senécal}

Volume 18, numéro 1, printemps 2009

Le corps. Sur le divan. Dans le fauteuil I

URI : https://id.erudit.org/iderudit/037722ar

DOI : https://doi.org/10.7202/037722ar

Aller au sommaire du numéro

Éditeur(s)

Revue Santé mentale au Québec

ISSN

1192-1412 (imprimé)

1911-4656 (numérique)

Découvrir la revue

\section{Citer ce document}

Senécal, F. (2009). La supervision en psychanalyse. Mutualité, assymétrie et refusement. Entrevue avec Lise Monette et Bernadette Tanguay. Filigrane, 18(1), 76-84. https://doi.org/10.7202/037722ar d'utilisation que vous pouvez consulter en ligne. 


\title{
La supervision en psychanalyse. Mutualité, assymétrie et refusement. Entrevue avec Lise Monette et Bernadette Tanguay
}

\author{
france senécal
}

\begin{abstract}
Liminaire
À la demande de Filigrane, j'ai rencontré Mesdames Lise Monette et Bernadette Tanguay, membres de la Société de psychanalyse de Montréal, dans le but de préparer une entrevue sur la thématique de la supervision en psychanalyse. Au fil de ces rencontres informelles autour de la question, certains thèmes ont émergé. À trois, nous avons tenté de donner forme à un objet construit à partir de nos interactions, mais sans en faire disparaître la mouvance d'une réflexion à vif. Les échanges ont été regroupés autour des thèmes que voici.
\end{abstract}

\section{Le superviseur comme tiers : position d'extériorité plutôt que de surplomb}

F. S. : Peut-on penser le superviseur comme un tiers, dans la mesure où il introduit une nouvelle écoute et brise le leurre de la relation duelle du supervisé avec son patient, voire même celle du supervisé avec son propre analyste, relations duelles qui peuvent être marquées par la toute-puissance?

L. M.: La supervision se situe, en un sens, en extériorité du lien entre l'analyste supervisé et son patient. Il s'agit d'une écoute, d'un regard sur la cure d'un patient; pointer le contre-transfert du supervisé c'est lui permettre de se le réapproprier, ce qui est différent pour moi que de s'immiscer dans le contretransfert ou de l'analyser. Ce qui, selon moi, appartient, à l'analyse personnelle.

B. T. : Mais il reste qu'en fait le supervisé peut fort bien s'approprier cette relation de supervision pour la faire jouer dans son analyse ou dans ses transferts. Quelque chose échappe toujours au travail du superviseur quand il ou elle accepte d'occuper cette place.

F. S. : Diriez-vous en cela qu'il y a une différence?

B. T.: J'ai parfois l'impression d'être utilisée par le supervisé comme lieu de transfert, comme surface de projection, mais non pas intemporelle et désincarnée. Position tierce vivante et incarnée. C'est très délicat, car on peut sentir cela, sachant que ce ne sera pas vraiment élaboré dans la supervision, à moins que cela ne fasse vraiment obstacle. Une forte idéalisation du superviseur peut parfois mener 
à une collusion qui bloque la pensée ; mais autrement, on laisse au supervisé cette appropriation qu'il se fait de nous, de nos propos.

F. S. : Il y a bien sûr des manifestations de l'inconscient dans la supervision mais diriez-vous que le travail qui s'y élabore se fait davantage au niveau conscient ou peut-être préconscient?

B. T. : On pourrait dire que la scène de l'inconscient est plutôt renvoyée à la scène de l'analyse.

F. S.: Cela vous place dans une position fort difficile et délicate, il me semble, celle de percevoir des enjeux inconscients importants et de ne pas «en jouer». Mais il s'agit aussi de faire en sorte que celui ou celle qui est en supervision puisse en faire quelque chose et dans sa propre analyse et dans son travail avec son patient. Il me semble que c'est là tout l'art de la supervision.

\section{Le conflit des écoutes}

F. S. : En quoi le conflit des écoutes qui survient entre superviseur et analyste supervisé peut-il vous sembler fécond?

L. M. : On peut penser que le conflit des écoutes crée, je ne suis pas tout à fait heureuse du terme qui vient, un relativisme des interprétations. Cela renvoie à l'écriture des cas clinique, par exemple, qui peut donner lieu à des types d'interprétations devenant mythiques, idéalisées, quasi prototypiques. La multiplication des écoutes - à travers plusieurs supervisions et celles de sa propre analyse accentue leur dimension hypothétique. Les interprétations devraient toujours être vues comme des hypothèses, de toute manière. Cela peut mener au conflit des interprétations et, au mieux, à une polyphonie des interprétations.

F. S. : Le conflit des interprétations pourrait alors devenir un espace de liberté pour le supervisé.

B. T. : Oui, une sorte d'espace de jeu, un espace où le supervisé pourrait se trouver comme analyste

F. S. : On pense certes à Winnicott : créer une espace transitionnel entre l'analyste et son patient, car vous devez percevoir parfois qu'il y a peu de «jeu», que c'est coincé entre le supervisé et son patient et que, pour tous deux, il s'agirait d'ouvrir. L. M. : Paradoxalement, je pense ce problème comme survenant surtout entre le supervisé et son analyste. La puissance de la projection et du transfert peut empêcher que cet espace se crée. On le voit bien dans les cas d'idéalisation massive. F. S.: Ce ne serait pas le but de la supervision, mais quelque chose qui arriverait de surcroît. Une chose précieuse qui, en bout de piste, aurait un effet sur le lien contre-transférentiel du supervisé avec son patient.

B. T. : Jeux de transferts comme des jeux de miroir; mais là, nous entrons sur le terrain du narcissisme et cela devient plus compliqué, d'autant plus qu'on se retrouve aussi du côté de l'identitaire et de la filiation.

\section{Filiation et formation}

F. S. : Depuis le début de l'entretien l'idée de filiation ne cesse de poindre... 
L. M. : J'aimerais introduire l'idée de supervision mutuelle, pas du tout au sens d'analyse mutuelle comme chez Ferenczi, mais, pour penser cette idée, nous avons besoin du concept d'asymétrie, en insistant sur le «a» privatif. Dans l'analyse, il est important que l'analyste ne soit pas à la même place que l'analysant, et il en va de même pour la supervision. Mais une position asymétrique ne veut pas dire une position hiérarchique, même si par ailleurs, dans l'institution il y a... hiérarchie.

B. T. : La question se pose alors de savoir comment le superviseur porte l'institution.

L. M. : Je crois qu'on peut penser à une asymétrie qui soit aussi mutuelle. Je voudrais qu'on sorte du lieu commun voulant qu'il n'y ait pas d'analyse à moins qu'il n'y ait un collègue et qu'on puisse concevoir que la supervision est le premier exercice de parole adressée à un collègue. Il y a asymétrie certes, mais la mutualité permet de ne pas infantiliser.

B. T.: J'aime bien l'idée qu'avait introduite France, celle d'un mouvement du superviseur qui fait advenir un collègue.

L. M. : En effet je crois qu'on ne peut faire advenir un collègue qu'à la condition de s'adresser au supervisé, dès le point de départ, non pas comme à un candidat mais comme à un collègue et ce n'est pas une coquetterie que de le formuler de cette manière.

F. S.: Il y a là une vision de la supervision bien différente de celle qui place le superviseur en position de surplomb, comme nous en parlait récemment J. C. Rolland.

B. T. : C'est ici toute la tentation pédagogique qui entre en jeu soit par notre lien à l'institution ou lorsque le superviseur se place en position d'autorité.

L. M. : Dans la supervision comme dans l'analyse, nous nous attaquons aux problèmes du transfert et du contre-transfert, en ce sens que, dans la supervision, nous sommes des collègues faisant face aux mêmes difficultés.

B. T. : Je dirais même que, en cette matière, l'expérience ne donne pas de longueur d'avance.

F. S.: Cette vision de la supervision se situe à une distance importante des autres conceptions. Il me semble qu'il s'agit ici d'un point tournant.

B. T. : Je crois en effet qu'il y a au moins deux philosophies de la formation; l'une va du côté de la filiation plus que de la formation comme telle, l'autre va du côté du travail entre collègues. En revanche il ne s'agit pas, dans cette deuxième, de dénier le rôle d'évaluateur. Le superviseur doit en effet assumer cette position, sinon il risque de sombrer dans la collusion, la séduction ou encore le copinage.

\section{Refusement $^{1}$}

F. S.: Il me semble que nous arrivons tout naturellement à la question que Lise Monette avait introduite dès le début, celle du refusement.

L. M. : J'aimerais en effet que nous nous arrêtions à la question du refusement. Il faudrait d'abord marquer la différence entre le refusement de l'analyste et celui du superviseur, qui permettrait au supervisé de tolérer l'ignorance, l'angoisse, 
l'ébranlement de ses repères. Alors que dans l'analyse, le refusement servirait davantage au déploiement du transfert.

B. T. : Ce n'est pas en effet la même visée. Dans la supervision il s'agit de tolérer l'angoisse du non-savoir ou de supporter un délai dans la compréhension.

L. M.: Cette question de l'angoisse me fait penser à la nécessité de créer un «cadre» à la supervision. J'ai presque envie de parler d'une zone de confort qui permette au supervisé de tolérer l'angoisse et de n'être pas d'emblée dans la maîtrise ou la compréhension.

F. S.: Cette idée d'un cadre de supervision me semble assez neuve; on n'en a pas souvent parlé en ces termes. Pourrait-on dire que le cadre ainsi conçu pourrait favoriser une sorte de régression, au sens de l'ébranlement des repères identificatoires?

L. M. : Je ne crois pas que cela s'applique vraiment aux supervisés, du moins en ces termes-là ; la régression a plus à voir avec le refusement de l'analyste dans la cure. Si cela arrive en supervision, c'est très rare.

F. S. : Il s'agirait alors plutôt de tolérer une position de non-savoir, quelque chose qui se joue davantage au niveau du préconscient que la régression elle-même.

L. M.: Oui, je pense que oui, Mais j'ajouterais que cela ressemble à un jeu de cartes où on ramasse la mise et puis on fait...

B. T. : ... une surenchère.

L. M.: Je vais peut-être faire une surenchère autour du refusement. Je me demande si à partir de la réflexion sur la maîtrise et le savoir, il ne faudrait pas parler de ce qui m'est cher, de la question de l'ascèse.

B. T. : Une position éthique.

L. M. : Je pourrais soutenir que s'il n'y avait pas chez le superviseur un refusement ou une mise en suspens de son propre savoir théorique...

B. T. : ... ou par rapport à sa propre expérience, du genre «moi je ferais ça »...

F. S. : ... mais le superviseur n'en a-t-il pas, une longueur d'avance ? Et cela renvoie aussi à une autre dimension : comment soutenir le travail du supervisé tout en sachant qu'il y aura évaluation de son travail, du moins pour les candidats à l'Institut?...

L. M. : J'aimerais réfléchir à cette expression : «longueur d'avance». Je n'en suis pas du tout certaine et, en ce sens, je partage l'opinion de Bernadette. Dans le cas de nouveaux patients, notre expérience nous sert parfois, mais dans d'autres cas, pas du tout. Je dirais qu'il en va de même pour la supervision. Les cas qu'on a vus nous servent en un sens, mais si l'on veut rester au plus près de ce qui se présente et du matériel apporté, pour permettre l'émergence d'un analyste en formation, en mouvement, je n'en suis pas du tout certaine.

B. T. : Il s'agit d'une sorte de paradoxe qui tient au fait que chacun est unique et exige une sorte de dé-saisissement...

F. S. : ... d'une position de savoir ; paradoxe de la position analytique elle-même...

L. M. : Oui

F. S.: Différemment d'une position scientifique ou les savoirs et les expériences contrôlées et vérifiées s'accumulent et se consolident. 
L. M. : On peut dire ça, mais en science aussi on peut piétiner.

B. T. : On peut penser à des profs qui ne renouvellent pas leur matériel et se répètent pendant des années. Il y a un piège dans le cumulatif.

F. S. : Si je puis me permettre, je suggère qu'il y a deux niveaux quand on parle de longueur d'avance. Elle existe certainement aux niveaux de la théorie et de l'expérience. On le sent vivement quand on commence une formation d'analyste à l'Institut, puisqu'on y vient toujours «d'ailleurs». On ne peut le nier: quand on vient, ou qu'on «sort» d'une discipline, que ce soit psychiatrie ou philosophie comme moi, et que l'on rencontre des superviseurs, on voit bien qu'ils ont une longueur d'avance sur soi. Toutefois dans le vif de la rencontre de supervision on sent moins qu'il y a longueur d'avance...

L. M. : Je me demande alors s'il ne faudrait pas poser cela autrement, en termes énergétique au sens métapsychologique... je vais faire un mini détour qui n'en est pas un. Par exemple, quelqu'un peut avoir eu des patients pendant un certain nombre d'années et n'avoir jamais éprouvé la force de l'intensité du transfert, de la névrose de transfert. J'utilise donc le terme «intensité» et c'est en ce sens que j'introduis la dimension énergétique. Parlerait-on alors d'expérience, non pas dans le sens cumulatif du terme, mais dans celui d'experiencing ? Les mots manquent ici pour parler du fait d'avoir rencontré et composé avec de telles intensités. Et je crois qu'avoir rencontré ces intensités-là dans la floraison de la névrose de transfert ou quand on la voit réapparaître en fin d'analyse, peut aider à diminuer l'angoisse.

F. S. : ... comme dirait Nathalie Zaltzman, d'avoir été «entamé » par le transfert.

B. T. : ... ou d'avoir vécu des régressions massives ou encore des crises majeures.

L. M. : C'est bien ce qui a à voir avec l'énergétique et l'intensité.

B. T.: On peut alors parler d'expérience, ce qui est autre chose que le cumul d'expériences qui consoliderait un savoir. Il s'agit, comme on dit, d'en avoir fait l'expérience, d'en avoir été marqué.

L. M. : Cela pourrait être la même chose par rapport à la théorie...

F. S. : En quel sens?

L. M. : Je pense que toi et moi, France, qui venons de philosophie, on peut voir des gens dans ce milieu qui ont une culture psychanalytique faramineuse. On peut prendre des cas extrêmes, parmi eux, qui n'auraient pas fait d'analyse personnelle ou que l'analyse personnelle aurait bien peu «entamés». Mais cette culture analytique fort impressionnante serait inutile, en un sens, parce qu'elle n'a pas été mise à l'épreuve de la clinique, du transfert et du contre-transfert, piège et mirage du contenu en lieu et place du repérage de la relation qu'il révèle. Enfin, je veux faire une différence entre une théorie mise à l'épreuve de la clinique versus une théorie qui fonctionne à partir de sa propre cohérence.

B. T. : L'attachement au contenu chez les cliniciens pourrait-il s'avérer un repli défensif tout autant que pourrait l'être une certaine manière d'avoir recours à la théorie, entravant par exemple la capacité de rêverie de l'analyste?

F. S. : Je me souviens d'avoir, pendant la formation et après, revisité des textes que j'avais longuement travaillés, voire même enseignés. À mon grand étonnement, 
l'effet du travail clinique et de la supervision me donnaient l'impression de les lire presque comme une première fois.

L. M. : Oui, j'irais jusqu'à dire que chaque fois qu'on lit un texte théorique, qui pour une raison ou une autre, ne vient pas rencontrer quelque chose de notre clinique, cette lecture demeure lettre morte. Mais il faut lire quand même parce qu'on ne sait pas quand cela finira par faire écho.

F. S.: Un texte littéraire peut aussi venir ouvrir une fenêtre dans du matériel clinique touffu et déroutant.

B. T. : Oui, bien sûr...

\section{Des manifestations de l'inconscient dans la supervision}

B. T. : La meilleure manière de parler de son patient est peut-être de le rêver, de se laisser traverser par son inconscient, d'aller du côté de l'association libre; mais on peut aussi écouter un supervisé parler de son patient du côté de l'association libre ; une sorte d'écoute flottante.

F. S.: On est loin du verbatim des séances qui peut être demandé au supervisé, rarement je crois à 1'IPM (Institut de psychanalyse de Montréal), mais ailleurs, oui.

L. M. : Notre conception de la supervision va complètement à l'encontre du verbatim comme de toute forme de compte rendu qui ferait fi de l'effet du refoulement sur l'écoute.

B. T. : Nous sommes plutôt du côté de l'écoute latente du supervisé.

L. M. : ... ce qui nous éloigne de l'interprétation «sauvage» de certains superviseurs. J'ai lu un analyste américain qui pensait que le transfert du supervisé sur le superviseur devait être interprété par le superviseur.

F. S. : Ne pourrait-on dire qu'en laissant tomber le verbatim, le type d'écoute que vous préconisez laisse entendre que c'est le supervisé qui tient le fil de l'analyse.

B. T. : Comme si on travaillait sur des morceaux choisis, mais qui, contrairement à ceux sur lesquels on travaillait au temps des collèges classiques, sont des morceaux non marqués par la censure.

F. S.: Morceaux choisis au sens d'objets partiels.

B. T. : Tiens, oui. Il s'agirait de prendre dans le matériel apporté des «morceaux », comme occasion de perlaboration autour d'un point de technique, de clinique ou de théorie.

L. M. : Faut-il le rappeler, il ne s'agit pas de faire du travail de synthèse, de construire du sens mais, à partir de fragments, de dégager les enjeux cliniques et théoriques, comme le souligne Bernadette.

B. T. : ... on n'est pas là pour intégrer les choses.

F. S. : ... ou articuler les séances les unes par rapport aux autres pour y repérer une «progression», par exemple, même s'il y a des moments de dégagement repérables, mais dont on ne sait jamais dans quel sens ils iront par la suite.

B. T. : Non justement, le suivi de la supervision n'est pas un travail systématique. Même si les supervisés nous le demandent souvent, de la même manière qu'ils 
nous demandent des références théoriques à propos du matériel apporté. Je respecte les mêmes principes, que les supervisés soient à l'IPM ou qu'ils viennent d'ailleurs. Certains supervisés nous disent même que cette manière de travailler est plus «mêlante» qu'autre chose. Je suis bien prête à le croire. On a parfois l'impression qu'on a un rôle désintégrateur. On introduit le flottement.

L. M. : Tu disais «être utilisée» par le supervisé; le message pourrait être: «apportez ce qui vous semble important et prenez de moi ce dont vous avez besoin »; c'est le supervisé qui fait le tri ; idéalement ce devrait être lui qui devrait en arriver à amorcer une théorie de son cas.

F. S. : Il ne s'agit donc pas d'appliquer des morceaux de théorie ou de règles techniques, même si en cours de supervision cela va surgir.

B. T. : En supervision, je puis me permettre de dire «ça me fait penser que », cela aurait pu me faire penser à tout autre chose, mais maintenant, voilà ce qui vient.

F. S.: On est au plus près de l'écoute analytique...

L. M. : Oui, sauf qu'en général, on ne fait pas part aussi activement aux patients de ce qui nous vient. Mais j'aimerais reprendre la question de la perlaboration et de l'élaboration. Il peut arriver que nous viennent des gens qui ne sont pas ou peu capables d'élaborer quoi que ce soit de ce qu'ils entendent de leurs patients et s'attendent à ce que nous élaborions à leur place, en quelque sorte ; on assiste à une sorte de mouvement de bascule.

F. S. : Déplacer l'écoute du supervisé...

L. M. : Oui, comme si on espérait que, par un élément de notre élaboration, à son tour le supervisé puisse élaborer, perlaborer autre chose. Ce serait comme dans un mouvement dialectique où une perlaboration peut entraîner chez le supervisé une relance de la perlaboration, ce qui pourrait nous éloigner de l'impasse pédagogique.

B. T. : Cela est parfois très difficile, parce que nous n'arrivons pas à nous appuyer sur le travail que certains supervisés n’ont pas réussi à faire sur leur monde intérieur ou parce que ce travail en est à ses débuts. Ainsi, ils sont peu travaillés, de l'intérieur par leurs propres mouvements transférentiels.

F. S. : Pourrait-on dire simplement qu'une supervision est d'autant féconde qu'elle peut s'appuyer sur l'analyse personnelle?

B. T. : Oh oui!

L. M. : Je le pense d'autant que parfois le travail de supervision peut amener des gens à entreprendre une analyse personnelle; c'est parfois ce que nous souhaitons pour sortir de l'impasse pédagogique de certaines supervisions.

B. T. : Dans le meilleur des cas, ils saisissent le travail en supervision comme un signal qu'ils doivent passer pour eux-mêmes dans le travail analytique, sinon on reste au niveau moïque, celui de la maîtrise et de la synthèse, on ne va pas très loin avec ça.

F. S.: Quand on ne peut s'appuyer sur l'analyse personnelle, cela peut sans doute être pénible tant pour le supervisé que pour le superviseur.

B. T. : Cela arrive... 
L. M. : On se retrouve alors démunies devant les manifestations de l'inconscient en supervision.

F. S. : Vous devez sentir que c'est comme un furet.

L. M. : Oui, c'est ça. Alors on reste au niveau manifeste; cela demeure très moïque. C'est très net: on est en présence de l'effet paralysant d'une idéalisation de la fonction analytique et, d'un surmoi, qui incite à mettre les freins tout le temps, à ne pas se laisser aller. Quelque chose qui veut forcer la compréhension et demeurer du côté de la maîtrise.

F. S. : Mais cela ne tiendrait-il pas à l'angoisse...

B. T. : ... d'être touché, déstabilisé.

F. S.: Cela peut-il à la limite compromettre le travail de supervision ?

B. T. : Je le crois. Des gens viennent parfois sur le bout des pieds, ont peur du temps que cela va durer, s'engagent en gardant la porte ouverte; pourtant il arrive qu'un véritable travail s'amorce.

L. M. : ... alors que d'autres peuvent venir «faire leurs heures » de supervision... F. S. : Comme à propos de la prison : «faire son temps »...

L. M. : Certains aussi découvrent, à leur corps défendant, leur incapacité à poursuivre plus loin. C'est très pénible.

B. T. : Pour eux et pour nous.

L. M. : On pourrait reprendre le terme d'aphanisis, à l'instar de Jones et Lacan, une nécessaire aphanisis du moi pour que puisse advenir une écoute analytique. Mais il faut aussi que le superviseur «lâche» son moi. Sinon on peut se retrouver avec des Moi en collusion. «Je te consolide, tu me confortes». Un délestage moïque est nécessaire de part et d'autre.

B. T. : Il faut aussi se demander comment penser les manifestations de l'inconscient en supervision; là aussi surgissent des lapsus, des ratés, des actes manqués. Ce n'est pas spécifique à la supervision, mais cela s'y produit aussi.

F. S.: Cela fait certes partie de la vie quotidienne des analystes en formation et la supervision en fait partie. Mais il me vient une pensée du Freud un peu plus tardif, disons que la supervision pourrait induire quelque chose de la dé liaison dans la mesure où l'on est dans le refusement de la consolidation, de la réassurance, de la maîtrise et des formes convenues de l'apprentissage.

B. T. : On peut alors voir l'espace où l'angoisse peut apparaître.

L. M. : Ah ! c'est une bonne piste. L'inconscient se manifestant alors dans le style de chaque analyste, fruit entre autres de la multiplicité des ses identifications partielles et de l'espace que lui assurent ses superviseurs loin de l'imitation du disciple.

F. S. : D'autant qu'on est dans le refusement de la consolidation, de la réassurance, de la maîtrise ou de l'apprentissage.

B. T.: Quand on parle de manifestation de l'inconscient en supervision avec un analyste, n'y a-t-il pas quelque chose qui se déploie sur le mode hystérique, qui se déplace de la cure à la supervision, déploiement de modes identificatoires dans une tentative de captation imaginaire? 


\section{4}

Filigrane, printemps 2009

L. M. : Est-ce que toute supervision ne porte pas en son sein un transfert latéral ? B. T.: Quand on a en analyse quelqu'un qui est en supervision, ne devrions-nous pas écouter quelque chose de ce côté?

L. M. : Je pense que c'est inévitable. Je vais être autobiographique pour un petit moment. Je me souviens de Julien Bigras, là je vais être au niveau du contenu manifeste avec toute sa charge, je le trouvais intrusif et je le sentais en compétition avec mon propre analyste. Je parlais en analyse, des reproches qu'il me faisait d'être trop théoricienne, mais aussi des compliments qu'il me faisait pour la même raison. Je m'en plaignais à mon analyste et enviais d'autres supervisés à qui leur analyste disait quoi faire, ou auxquels il donnait des indices assez clairs. Le mien ne le faisait jamais et je n'arrêtais pas de lui reprocher. J'enviais, par exemple, une collègue pour ce que Scott lui donnait. Ah le refusement... Mais cela a peut-être peu à voir avec la supervision; je suis en train de penser que je suis allée voir Scott pour qu'il me donne ce qu'il avait donné à cette collègue !...

Un superviseur pour l'amour!!!

\section{Note}

1. Cette notion renvoie à l'exigence pour l'analyste dans la cure, d'une ascèse qui permet une neutralisation des charges pulsionnelles engendrées par le processus lui-même et qui dégage, pour le patient, son propre espace d'élaboration. 